\title{
Rectal prolapse in a child with cow's milk allergy
}

\author{
Sandra Lucarelli • Giovanni Di Nardo • \\ Simone Frediani • Tullio Frediani • Denis A. Cozzi • \\ Salvatore Cucchiara
}

Accepted: 26 February 2009/Published online: 11 March 2009

(C) Springer-Verlag 2009

\section{Dear Editor:}

A child aged 3 years 4 months, breast-fed for the first 3 months of life, with a clinical history of persistent constipation unaffected by treatment with laxatives presented recurrent episodes of complete rectal prolapse after the introduction of cow's milk into his diet. The rectal prolapse disappeared after this substance was eliminated from the diet. There was no family history of atopy.

Before admission for observation, the child had presented eight episodes of rectal prolapse in the space of 3 years (the last two a fortnight before being admitted). While the results of prick tests carried out to ascertain whether the constipation was a symptom of food allergy proved negative, a patch test with cow's milk was positive. Total IgE were within the normal values and there were no specific IgE. The sweat test was negative. Parasitic infestation and coeliac disease were excluded. Colonoscopy revealed disorders of the mucosa compatible with the diagnosis of lymphoid hyperplasia, as confirmed by histologic examination of the biopsy specimens. This finding, which proves frequent in allergic enteropathies, suggested that an elimination diet for cow's milk protein might prove effective.

\section{S. Lucarelli $(\varangle) \cdot$ G. Di Nardo $\cdot$ S. Cucchiara}

Pediatric Gastroenterology Unit, Sapienza University of Rome,

Azienda Policlinico Umberto I, Viale Regina Elena, 324,

00161 Rome, Italy

e-mail: sandra.lucarelli@uniroma1.it

\section{S. Frediani $\cdot$ D. A. Cozzi}

Pediatric Surgery Unit, Sapienza University of Rome,

Azienda Policlinico Umberto I,

Rome RM 00161, Italy

\section{T. Frediani}

Pediatric Allergology Unit, Sapienza University of Rome, Azienda Policlinico Umberto I,

Rome RM 00161, Italy
The constipation disappeared over the following 6 weeks and there was no recurrence of rectal prolapse. When the patient was orally exposed to fresh cow's milk as a check on the diagnosis, persistent constipation reappeared within 7 days together with complete rectal prolapse. The child was placed on a diet free of cow's milk protein. Follow-ups carried out after 2 and 6 months in the absence of pharmacological treatment confirmed the regularity of the intestinal function and the absence of rectal prolapse.

Rectal prolapse in pediatrics has its highest incidence in infancy and is seldom seen in industrialized countries. The prolapse may involve only the mucosa (mucosal prolapse) or all layers of the rectum (complete prolapse or procidentia). It is sometimes detected by the child's parents and brought urgently to medical attention but has usually reduced spontaneously by the time they reach the practitioner's office. Rectal prolapse should be viewed as a symptom of an underlying condition rather than a separate illness. Potential causes are increased intra-abdominal pressure, diarrheal and neoplastic diseases, malnutrition, and conditions conducive to pelvic floor weakness.

The strong association with cystic fibrosis makes the sweat test mandatory for infants and children with recurrent rectal prolapse. Chronic constipation is a common condition in pediatrics, its treatment is far from being satisfactory. More than one thrird of patients are still symptomatic 5 years after diagnosis and constipation may persist in one third into early adulthood. Attention has been drawn in recent years to a relationship between cow's milk protein allergy and chronic constipation.

This case, the second reported in the literature, appears to bear out the hypothesis that allergy to cow's milk protein can cause severe constipation combined with complete rectal prolapse through a delayed type of immunological mechanism, as suggested by the positive result of the patch test in the absence of immunological mechanisms of the reaginic type. 